A PAP in the last year before this study was available in $30 \%$ of cases.

The correlation of screening techniques with the three methods proposed: diagnostic cytology, capture hybrid and colposcopy was made in 169 women

Prevalence of HPV infection was 39\% (104 positive/165 negative).

Correlation between Cyto-colposcopic and Hybrid Capture test was:

hybrid capture $(-)$ cytology/colposcopy $(+)=2 / 169$

hybrid capture $(+)$ cytology/colposcopy $(-)=15 / 169$

hybrid capture $(+)$ cytology/colposcopy $=32 / 169$

hybrid Capture (-) cytology colposcopy $(-)=84 / 169$

hybrid Capture $(+)$ indeterminate cytology and colposcopy $(-)=19 / 169$

hybrid Capture (-) indeterminate cytology and colposcopy $(-)=17 / 169$

Conclusions Despite that it was not a large sample, the negative predictive value of hybrid captures for HPV detection was $98 \%$ in this population.

We recommend the incorporation of the hybrid capture as screening technique in all women with HIV regardless of the age and all those with negative cytology and positive hybrid capture for the early detection of cervical lesions.

\section{P3.048 HIV IN CHILDREN IN A GENERAL POPULATION SAMPLE IN SOUTHERN AFRICA: PREVALENCE, CAUSES AND EFFECTS}

doi:10.1136/sextrans-2013-051184.0508

'E L Pufall, 2,1C Nyamukapa, ' J W Eaton, ${ }^{2} \mathrm{R}$ Mutsindiri, ${ }^{2} \mathrm{G}$ Chawira, ${ }^{2} \mathrm{~S}$ Munyati, 'L Robertson, 'S Gregson. 'Imperial College London, London, UK; ${ }^{2}$ Biomedical Research and Training Institute, Harare, Zimbabwe

Background The epidemiology of HIV in children in sub-Saharan Africa remains poorly understood. To help address this gap we describe the prevalence, causes and effects of HIV in children (aged 2-14 years) and adolescents (aged 15-17 years) in a southern African population.

Methods General population survey of 3,390 children and 2,130 adolescents in Manicaland, eastern Zimbabwe. Data on possible socio-demographic correlates of HIV prevalence, sources of infection, and effects of HIV infection in children were analysed using multi-variable logistic regression. The contribution of horizontal transmission was assessed by investigating the survival and HIV infection status of mothers of infected children.

Results HIV prevalence was $2.15 \%(73 / 3,390)$ in children and $1.97 \%(42 / 2,130)$ in adolescents. Infection levels did not differ by sex, age, socio-economic status, location or religion. The mothers of most infected children were either deceased or HIV-positive which is consistent with MTCT being the primary mode of infection, but 9/73 infected children and 4/42 infected adolescents had surviving uninfected mothers. Infected children were more likely than uninfected children to be malnourished $(21.6 \%$ versus $9.9 \%, p=0.006)$ and stunted $(42.0 \%$ versus $30.5 \%, p=0.03)$. HIV infection was not correlated with physical or psychological ill-health in children, but infected adolescents were more likely to report episodes of illness than their uninfected counterparts $(p=0.003)$.

Conclusion Childhood HIV infection in Zimbabwe is due primarily to MTCT and is associated with poor physical development.

\section{P3.049 HIV/AIDS IN A PUERTO RICAN WOMEN POPULATION: PSYCHOSOCIAL, RISK BEHAVIOR AND LIFESTYLES}

doi:10.1136/sextrans-2013-051184.0509

D M Fernandez-Santos, A M Mayor, E Santiago, E Rios-Olivares, R F Hunter-Mellado. Universidad Central del Caribe, Bayamon, PR, United States
Background This study describes psychosocial, risk behaviour and lifestyle features of HIV/AIDS women in Bayamón, PR. It also compares the psychosocial, behavioural and lifestyle profile changes of this cohort according to their entry year to the registry (2000-2010). Methods Baseline data was analysed from a prospective survey of HIV/AIDS-infected women reported to the HIV Central Registry at the Universidad Central del Caribe, RCMI Program from 2000 to 2010. The study group was composed of 499 adult women, with AIDS or HIV infection reported upon arrival at the University Hospital Ramon Ruiz Arnau or the Bayamon Regional Immunology Clinic. Statistical analysis included frequencies, percentages, Chisquare, Chi Square for linear trend and Fisher Exact test.

Results A large proportion of women were under 46 years $(71.4 \%)$. Most reported not having graduate studies (98.8\%), being unemployed (70.9\%), not having a partner in the last year (57.0\%) and having children $(88.0 \%)$. The most frequent sexual risk behaviour was heterosexual activity (98.8\%), where $71.3 \%$ reported heterosexual activity in the last year and $14.4 \%$ reported prostitution activity. Risky lifestyles reported by women included: smoking tobacco $(64.7 \%)$, use of alcohol (38.3\%), use of psychoactive substances $(36.3 \%)$ and intravenous drug usage (IVDU) (36.3\%). Most frequent psychosocial factors reported were: episodes of depression (82.2\%), episodes of impulsivity (55.4\%), isolation (44.6\%), suicide attempt $(23.7 \%)$, time served in prison $(20.3 \%)$, voluntary miscarriage $(17.7 \%)$ and having been sexually assaulted (7.1\%). An increasing linear trend $(p<0.05)$ was observed among proportions of women that reported using psychoactive substances, attempting suicide and having been sexually assaulted.

Conclusions Women reported a wide spectrum of risky psychosocial, behavioural and lifestyle activities. Increasing changes among these were observed over the last decade. This effort was made possible by NIH grant number 8G12MD007583 and 8U54MD007587 from the National Institute on Minority Health and Heath Disparities.

\section{P3.050 HIGH-RISK UROGENITAL HPV INFECTIONS IN PARAMARIBO, SURINAME: PREVALENCE AND RISK FACTORS AMONG ETHNIC DIVERSE WOMEN}

doi:10.1136/sextrans-2013-051184.0510

IJ J van der Helm, ${ }^{2} \mathrm{D}$ Geraets, ${ }^{3} \mathrm{~A}$ Grunberg, ${ }^{2,4 \mathrm{~K}}$ Quint, ${ }^{5} \mathrm{~L}$ Sabajo, ${ }^{1,6,7 \mathrm{H}}$ de Vries. 'Health Service Amsterdam, Amsterdam, The Netherlands; ' $2 D D L$ Diagnostic Laboratory, Rijswijk, The Netherlands; ${ }^{3}$ Dept. of Public Health Ministry of Health Suriname, Paramaribo, Suriname; ${ }^{4}$ Department of dermatology LUMC, University of Leiden, Leiden, The Netherlands; ${ }^{5}$ Dermatological Service, Ministry of Health Suriname, Paramaribo, Suriname; ${ }^{6}$ Academic Medical Center, University of Amsterdam, Amsterdam, The Netherlands; ${ }^{7}$ Centre for Infectious Disease Control, National Institute of Public Health and the Environment, Bilthoven, The Netherlands

Background and Aim: Cervical cancer is caused by high-risk (HR) Human Papilloma Virus (HPV) infections. The mortality rate of cervical cancer in Suriname is $8 / 100.000$ per year. Current HPV vaccinations protect against cancers induced by HR-HPV 16 and -18 , and are not yet available in Suriname. We estimated prevalence of, and risk factors for urogenital HR-HPV infections among women from five major ethnic groups in Paramaribo, Suriname in a pre-vaccination era.

Methods Between July 2009 and February 2010, women aged $\geq 18$ years were recruited at a Family Planning clinic and STI clinic. Vaginal swabs were collected and general HPV detection was performed using the highly sensitive broad-spectrum SPF10 PCR and DNA enzyme immunoassay (DEIA). Subsequently, DEIA-positive samples were genotyped by the LiPA25 reverse hybridization assay (Labo Bio-medical Products, The Netherlands), targeting 25 different genotypes. Logistic regression analysis was used to identify determinants of HPV infection. 
Results 1001 women were included and tested for HPV of whom 584 (58\%) were DEIA positive. Of the positive samples 472 (81\%) could be typed by LiPA25. HR-HPV types were detected in 303 (30\%) of all samples. HPV type 52 was the most prevalent genotype ( $\mathrm{n}=79 ; 8 \%$ ). Of the HR-HPV, 46 (15\%) had only HR-HPV types 16 and/or 18, covered by available vaccines. Adjusted for age the following independent associations with HR-HPV were found; $\geq 2$ recent partners $(\mathrm{OR}=1.5,95 \% \mathrm{CI}=1.1-2.2)$, Chlamydia trachomatis co-infection $(\mathrm{OR}=2.3,95 \% \mathrm{CI}=1.5-3.5)$ and ethnic group $(\mathrm{OR}=2.1,95 \% \mathrm{CI}=1.4-3.1$ for Creole; $\mathrm{OR}=2.0,95 \% \mathrm{CI}=1.2-3.3$ for Maroon; $\mathrm{OR}=2.8,95 \% \mathrm{CI}=1.8-4.4$ for Mixed race compared to Hindustani).

Conclusions HR-HPV 52 is the most prevalent type in Suriname and HR-HPV is not equally distributed among ethnic groups. $85 \%$ of HR-HPV infections involve types that are not covered by the 2 currently available vaccines against cervical cancer. These data provide a basis for possible shifts in HPV genotype prevalence following vaccination.

\section{P3.051 PRE-VACCINATION PREVALENCE OF INFECTIONS WITH 25 LOW-RISK HUMAN PAPILLOMAVIRUS (HPV) TYPES AMONG 1000 SLOVENIAN WOMEN SCREENED FOR CERVICAL CANCER IN 2010}

doi:10.1136/sextrans-2013-051184.0511

V Učakar, ${ }^{2} \mathrm{M}$ Poljak, ${ }^{2} \mathrm{~A}$ Oštrbenk, "I Klavs. ${ }^{1}$ National Institute of Public Health, Ljubljana, Slovenia; 'Institute of Microbiology and Immunology, Faculty of Medicine, University of Ljubljana, Ljubljana, Slovenia

Background Our objective was to estimate pre-vaccination prevalence of cervical infections with 25 non-high-risk human papillomavirus (HPV) types, referred to as low-risk HPV (LR-HPV) types, regardless of and without the coexistence of high-risk HPV (HRHPV) types among Slovenian women 20-64 years old screened for cervical cancer.

Methods 1000 cervical specimens randomly selected from 4455 specimens collected in the Slovenian HPV prevalence survey with a nationally wide coverage in 2010 were tested with Linear Array HPV Genotyping Test.

Results Prevalence of cervical infections with any of the 25 LRHPV types was $10.0 \%$ (95\% CI: $8.1 \%-11.9 \%)$ and with exclusively LR-HPV types $4.5 \%$ (95\% CI: $3.2 \%-5.8 \%$ ). Prevalence of infections with any LR-HPV types among women with normal cytology was $8.8 \%$, with atypical squamous cells of undetermined significance (ASC-US) $30.4 \%$, with low grade squamous intraepithelial lesions (LSIL) $60.0 \%$, and with high grade squamous intraepithelial lesions (HSIL) $7.7 \%$. LR-HPV types without coexisting HR-HPV types were found in $4.0 \%$ of women with normal cytology, $26.1 \%$ with ASCUS, $6.7 \%$ with LSIL, and none with HSIL. Infections with exclusively HPV 6 or HPV 11 were not found.

Conclusions LR-HPV type cervical infections without coexisting HR-HPV infections were common among Slovenian women screened for cervical cancer with ASC-US, while rare in those with pathological cytology result. With respect to organised cervical cancer screening programme in Slovenia our results suggest that HRHPV testing based triage that complements the follow-up cytology in the Slovenian cancer screening programme since 2010, will contribute to some reduction in the Slovenian NCCSP follow-up burden, especially among women with ASC-US and some decrease in related patient anxiety.

\section{P3.052 FREQUENCY OF MULTIPLE HPV GENOTYPES IN WOMEN FROM VOJVODINA, SERBIA}

doi:10.1136/sextrans-2013-051184.0512

V S Milosevic, Gordana Kovacevic, Ivana Hrnjakovic-Cvjetkovic, Jelena Radovanov, Ivana Elez, Aleksandra Patic. Institute for public health of Vojvodina, Novi Sad, Serbia
Background Persistent infection with high-risk (HR) human papillomavirus (HPV) types is necessary for cervical cancer development. The risk is much higher for women infected with multiple genotypes of the human papillomavirus (HPV). The aim of this study was to evaluate the frequency of single and multiple HPV infection in relation to age and results of Pap smear cytology.

Methods From January to December 2012, frequency of HR HPV was analysed among 398 women from Vojvodina. In determination of the presence HPV DNA commercial HR HPV Real-TM kit (Sacace Biotechnologies, Italy) were applied. PCR was performed and products were detected using ABI 7500 Real-Time PCR instrument.

Results The overall prevalence of HPV infection among studied women was $61.3 \%$. Single HPV genotypes were found in 150 (65.3\%) and multiple genotypes in 94 (34.7\%) of 244 HPV DNA positive samples. The most frequent genotype was HPV 16, followed by HPV 31, 51.52 and 18. Proportion of multiple HPV infection decreased significantly according to age and severity from $48.9 \%$ in women under 30 years of age, $39.7 \%$ in women from 30 to 40 years of age, $21.3 \%$ in women older than 40 . Among all women with normal cytology multiple HPV infections were found in (22.4\%), (34.9\%) among women with ASCUS cytology, (17.0\%) in LSIL cytology and (17.9\%) in HSIL cytology.

Conclusion In the present study, we observed a very high prevalence of multiple $(22.4 \%)$ of HPV infection among women with normal cytology and (34.9\%) in women with indeterminate Pap test, as ASCUS cytology. Sensitive and specific detection methods, as well as, real-time PCR genotyping HPV are required and helpful for triage of women with ASCUS cytology.

\section{P3.053 IN WHICH CIRCUMSTANCES DOES HPV ENTER PARTNERSHIPS? A DYADIC-LEVEL ANALYSIS}

doi:10.1136/sextrans-2013-051184.0513

'A N Burchell, ${ }^{2} \mathrm{~A}$ Rodrigues, ${ }^{3} \mathrm{~F}$ Coutlee, ${ }^{2} \mathrm{P}$ Tellier, ${ }^{2} \mathrm{~J}$ Hanley, ${ }^{2} \mathrm{E}$ L Franco. ${ }^{1}$ Ontario HIV Treatment Network, Toronto, ON, Canada; ${ }^{2}$ McGill University, Montreal, OC, Canada; ${ }^{3}$ Universite de Montreal, Montreal, OC, Canada

Background Understanding patterns of human papillomavirus (HPV) infection in partnerships is essential in exploring transmission of HPV in sexual networks. Risk factors for HPV infection have yet to be explored at the dyad level. We studied features that predict presence of HPV in a new sexual partnership.

Methods We analysed data from the HITCH Cohort Study of recently-formed couples. Women aged 18-24 attending university/ college in Montreal, Canada and their male partners were recruited in 2006-10. Self-collected vaginal swabs and clinician-obtained swabs from the penis and scrotum were tested for DNA of $36 \mathrm{HPV}$ types. We analysed baseline data from 479 couples. HPV in a partnership was defined as the presence of 1 or more HPV types in either or both partners. We used Poisson regression to calculate prevalence ratios with 95\% confidence intervals for candidate risk factors.

Results Most women were unvaccinated (88\%). 67\% of partnerships harboured HPV. For $49 \%$ both partners were HPV + . Detection was associated with the combined total of the male's and female's lifetime partners; from $27.5 \%$ among couples who jointly had no more than 4 partners to $94.2 \%$ among couples with $>20$. Couples reporting concurrent partners were 2.8 times (95\% CI 1.7-4.5) more likely to have HPV compared to those with a 12-month gap since the last extra-dyadic partner but this effect disappeared after adjustment for number of partners. Couples who always or frequently used condoms with their previous partner(s) were 29\% (95\% CI 9-45\%) less likely to have HPV after accounting for number of partners and gap length/concurrency.

Conclusions Number of extra-dyadic partners (past or concurrent) predicts the likelihood of HPV in a partnership. Condoms may have some impact on limiting spread of HPV, although protection 